J. Clin. Chem. Clin. Biochem.

Vol. 16, 1978, pp. 299-305

\title{
Ausarbeitung einer Methode zur quantitativen Proteinbestimmung mit Hilfe eines Laser-Nephelo- meters im Routinelabor
}

Von A. Conrad, J. Schürmann, F. H. Kreutz

Zentrallaboratorium Stadtkrankenhaus Kassel und

\section{A. Sieber}

Behringwerke AG, Marburg/Lahn

(Eingegangen am 20. August/28. Dezember 1977)

Zusammenfassung: Die Reaktionsbedingungen der quantitativen Bestimmung von IgG, IgA und IgM im LaserNephelometer werden untersucht. Daraus-ergibt sich ein einfaches Arbeitsschema. Die Methode wird mit der radialen Immundiffusion verglichen und statistisch ausgewertet.

\section{Elaboration of a method for the quantitative determination of proteins by laser nephelometry in the clinical routine laboratory}

Summary: The reaction conditions for the quantitative determination of IgG, IgA and IgM in the laser nephelometer were investigated. An easy working scheme was devised. The method was compared with radial immunodiffusion, and it was statistically evaluated.

\section{Einführung}

Proteine lassen sich mit Hilfe von Laser-Streulichtmessungen an Antigen-Antikörper-Komplexen quantitativ bestimmen $(1,2,3)$. Der Einsatz eines Lasers als Lichtquelle für nephelometrische Proteinbestimmungen bringt bestimmte Vorteile mit sich. Die spezielle Geometrie des Laser-Strahles erlaubt, das in Vorwärtsrichtung an den Antigen-Antikörper-Komplexen gestreute Licht unter sehr kleinen Winkeln zu messen.

Die Intensität dieses Streulichtes ist gerade in Vorwärtsrichtung besonders groß, was eine hohe Empfindlichkeit dieser Meßanordnung zụr Folge hat (1).

Es kommt darauf an, daß man den direkten Lichtstrahl vollständiğ ausblendet, was bei einer herkömmlichen Lampe wegen der fehlenden Parallelität des Lichtes auf größere Schwierigkeiten stößt.

Ferner sendet der Laser Licht mit einem zeitlich sehr konstanten Strahlungsfluß aus, was eine gute Reproduzierbarkeit der Messungen zur Folge hat.

Ziel der Arbeit ist es zu untersuchen, ob ein LaserNephelometer auch für routinemäßige Proteinbestimmungen Vorteile bietet.

\section{Material und Methoden}

\section{Gerät}

Die Versuche wurden mit dem Behring Laser-Nephelometer (Behringwerke AG) durchgeführt. Das Gerät arbeitet mit einem Helium-Neon-Laser, Wellenlänge $632,8 \mathrm{~nm}$, Leistung $4 \mathrm{~mW}$. Aufgrund der geschilderten Eigenarten der Meßanordnung (1) benötigt das Gerät keinen Photomultiplier, weswegen auch die Umschaltung auf verschiedene Meßbereiche entfallt. Abbildung 1 zeigt eine schematische Darstellung der Meßanordnung.

Die Streulichtintensität wird bei dem Gerät über ein Digitalvoltmeter abgelesen. Das Instrument verfügt über eine Meßbereichsanzeige von $0,00 \mathrm{~V}-19,99 \mathrm{~V}$.

\section{Reagenzien}

Zur Erstellung der Referenzkurven wurde das Protein-StandardSerum LN der Behringwerke eingesetzt. Es handelt sich hierbei um ein stabilisiertes gepooltes Humanserum von gesunden Spendern. Spezielle für die Laser-Nephelometrie gẹ̈eignete Antiseren (LN-Antiseren, Behringwerke AG) zur Bestimmung von IgG, IgA und IgM wurden verwendet.

Als Verdünnungsmittel für Standardserum, Patientenseren und Antiseren diente staubfreie physiologische Nảtriumchloridlösung. Die NaCl-Lösung und die Antiserum-Vèrdünnung wurden vor Gebrauch filtriert (Millex Disposable Filter Units, Millipore GmbH, Neu Isenburg). Versuchsweise wurden phosphatgepufferte $1 / 15 \mathrm{~mol} / 1$ Natriumchloridlösung, pH.7,2, und Polyethylenglykol, Molekulargewicht 6.000 (PEG 6.000, Serva, Heidelberg) eingesetzt. Polyethylenglykol wurde in einer Konzentration von $40 \mathrm{~g} / 1$ phosphatgepufferter NaCl-Lösung verwendet. 


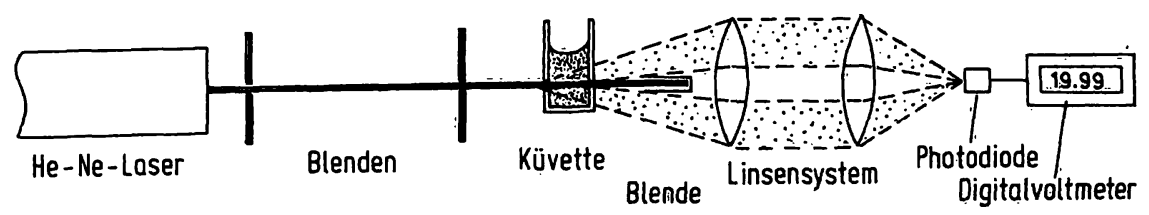

Abb. 1. Strahlengang des Laser Nephelometers.

Tab. 1. Verdünnungsschema

\begin{tabular}{llllll}
\hline & $\begin{array}{l}\text { Verdünnungen des Protein- } \\
\text { Standard Serum LN }\end{array}$ & $\begin{array}{l}\text { Verdünnung der } \\
\text { Antiseren }\end{array}$ & $\begin{array}{l}\text { Verdünnung der } \\
\text { Patientenseren }\end{array}$ & $\begin{array}{l}\text { Volumen der } \\
\text { Standard oder } \\
\text { Probenverdünnung }\end{array}$ & $\begin{array}{l}\text { Volumen der Anti- } \\
\text { serumverdünnung }\end{array}$ \\
\hline IgG & $\begin{array}{l}1: 20,1: 40,1: 80,1: 160, \\
1: 320,1: 640\end{array}$ & $1: 5$ & $1: 101$ & $10 \mu 1$ & $200 \mu 1$. \\
IgA & $\begin{array}{l}1: 20,1: 40,1: 80,1: 160, \\
1: 320,1: 640\end{array}$ & $1: 5$ & $1: 101$ & $100 \mu l$ & $200 \mu 1$ \\
IgM & $1: 10,1: 20,1: 40,1: 80$, & $1: 5$ & $1: 101$ & $100 \mu l$ & $200 \mu 1$ \\
\hline
\end{tabular}

Reaktion und Messung der Proben erfolgte in Einwegküvetten aus Kunststoff mit spezieller Eignung für die Laser-Nephelometrie (LN-Küvetten, Behringwerke AG, W. Sarstedt).

Für Korrelationsuntersuchungen zwischen radialer Immundiffusion und Laser-Nephelometrie wurden Tri-Partigen-Immundiffusionsplatten (Behringwerke) und die entsprechenden TriPartigen-Standards eingesetzt.

Die Ablesung der Platten erfolgte mit dem Partigen-Meßprojektor (Behringwerke AG).

\section{Proben}

Zur Untersuchung gelangten 80 Patientenseren aus dem täglichen Anfall des Zentrallabors einer Großklinik (Stadtkrankenhaus Kassel). Es wurde darauf geachtet, daß keine stark getrübten Seren ohne weitere Vorbehandlung zur Messung gelangten.

\section{Durchführung der Bestimmungen}

\section{Nephelometrie}

Das Standard-Serum wurde in einer geometrischen Verdünnungsreihe von 1: 10 bis $1: 640$ mit NaCl-Lösung eingesetzt. Mit diesen Verdünnungen ließen sich die Referenzkurven der drei o. a. Proteinbestimmungen aufstellen. Die Antiseren wurden bei allen Bestimmungen mit $\mathrm{NaCl}$-Lösung $1: 5$ verdünnt. Die Patientenseren wurden mit $\mathrm{NaCl}-\mathrm{Lösung} 1: 101$ vorverdünnt. Die Blindwerte der leeren Küvetten wurden bestimmt und von den Meßwerten subtrahiert.

Bei der IgG-Bestimmung reagierten $10 \mu l$ der Standard- bzw. Serum-Verdünnung mit $200 \mu 1$ Antiserumverdünnung. Bei der IgA- und IgM-Bestimmung betrug das Serumvolumen jeweils $100 \mu \mathrm{l}$ und das Antiserumvolumen $200 \mu \mathrm{l}$. Die Mischung der Lösungen erfolgte durch leichtes Schütteln der Küvetten. Schaumodèr Blasenbildung wurden vermieden. Die Reaktionszeit betrug eine Stunde.

Die Referenzkurven wurden auf doppeltlogarithmischem oder linearem Millimeterpapier gezeichnet.

Die Tabelle 1 gibt das Verdünnungsschema wieder, nach dem die Proteinbestimmungen durchgeführt wurden.

Die Vergleichsmessungen mit Hilfe der radialen Immundiffusion wurden gemäß den Vorschriften des Herstellers durchgeführt.
Versuchsweise wurde eine Auswertung mit einem Tischrechner, HP-Calculator 91, durchgefürt (Tab. 2). Es zeigte sich, daß die Referenzkurven einfachen ln-Funktionen entsprachen, deren Berechnung über eine „logarithmische“ Kurvenpassung möglich war. Das verwendete Programm errechnete die Anpassung der In-Funktion $\mathrm{y}=\mathrm{a}+\mathrm{b} \ln x$ nach der Methode $\bar{c}$ ir kleinsten Quadrate an $n$-Datenpaaren $\left(x_{j}, y_{j}\right), i=1,2, \ldots, n$ mit $y>0$. Da negative Meßwerte nicht möglich sind, ist $b>0$ gegeben.

Die Koeffizienten a, b müssen wie folgt berechnet werden:

$$
\begin{aligned}
& \mathrm{b}=\frac{n \Sigma \mathrm{y}_{\mathrm{i}} \ln \mathrm{x}_{\mathrm{i}}-\Sigma \ln \mathrm{x}_{\mathrm{i}} \Sigma \mathrm{y}_{\mathrm{i}}}{\mathrm{n} \Sigma\left(\ln \mathrm{x}_{\mathrm{i}}\right)^{2}-\left(\Sigma \ln \mathrm{x}_{\mathrm{i}}\right)^{2}} \\
& \mathrm{a}=\frac{\Sigma \mathrm{y}_{\mathrm{i}}-\mathrm{b} \Sigma \ln \mathrm{x}_{\mathrm{i}}}{\mathrm{n}} \cdot \begin{array}{l}
\mathrm{y}_{\mathrm{i}}=\text { Meßwerte in } \mathrm{V} \\
\mathrm{x}_{\mathrm{i}}=\text { Konzentrationen } \mathrm{g} / \mathrm{l}
\end{array}
\end{aligned}
$$

Mit Kenntnis dieser beiden Konstanten läßt sich zu jedem y-Wert der dazugehörige $\mathrm{x}$-Wert berechnen.

Eingabeschema für die Wertberechnung $x_{i}=e^{\frac{y_{i}-a}{b}}$ Eingabewerte Operationen

Ergebnisse

$\mathbf{y}_{\mathbf{i}}$

$$
\text { RCL } 2-\operatorname{RCL} 1 \div \mathrm{e}^{\mathrm{x}} \quad \mathrm{x}_{\mathrm{i}}
$$

Diese Vorgehensweise ist nur gültig, wenn man voraussetzen kann, daß die Linearisierung $y=a+b \ln x$ möglichst fehlerfrei ist. Dies war in unserem Falle, wiè Abbildung 2, auch stellvertretend für IgM und IgG zeigt, gegeben.

Tab. 2. Eingabeschema für die Berechnung der Referenzkurven mit dem Rechner HP 91

\begin{tabular}{lll}
\hline Eingabewerte & Operationen & Ergebnisse \\
\hline$y_{i}$ & Enter $\uparrow$ & \\
$x_{i}$ & $\ln \Sigma+$ & \\
& LR STO 1 & b \\
& $x_{\uparrow} y_{\text {STO 2 }}$ & a \\
\hline
\end{tabular}




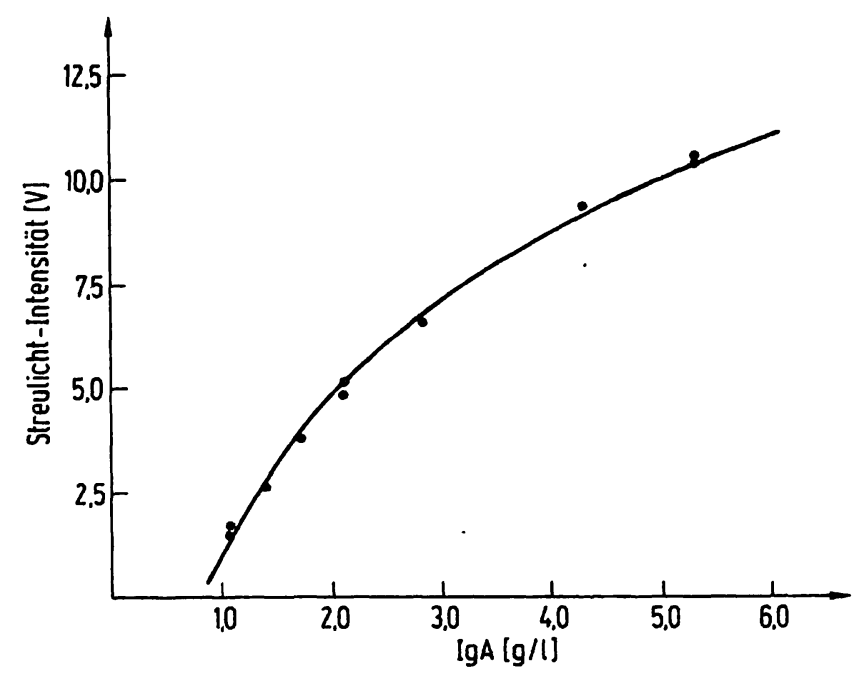

Abb. 2. Logarithmische Kurvenanpassung der Referenzkurve für $\lg A, y=5,77 \ln x+0,87$

\section{Ergebnisse}

\section{Blindwerte}

Die Messung der Küvettenblindwerte ergab, daß die Schwankungen der Eigenstreulichtintensitäten von Küvette zu Küvette sehr gering waren (VK $<10 \%)$. Die mittlere Schwankung der Küvettenblindwerte betrug etwa $0,5 \%$ der bei Proteinbestimmungen gemessenen Streulichtsignale von etwa $10 \mathrm{~V}$.

Die Blindwerte der verwendeten Antiserumverdünnungen lagen unter den Blindwerten der Küvette.

Die Blindwerte der Patientenseren wurden in drei Kategorien unterteilt:

Klare Seren, die bei visueller Prüfung keine Trübung erkennen ließen, zeigten in den entsprechenden Arbeits- verdünnungen Blindwerte, die unter denen der Küvetten lagen. Infolge der hohen Verdünnungen zeigten auch Seren mit leichter Trübung vernachlässigbare Blindwerte. Erst bei stark trüben Proben traten auch in den Endverdünnungen Streulichtsignale von mehr als $0,5 \mathrm{~V}$ auf.

Eine Korrektur durch Subtraktion der Blindwerte stark trüber Seren von den Meßwerten führte zu widersprüchlichen Ergebnissen.

Die Filtration trüber Seren über Millex-Einwegfilter brachte nicht in allen Fällen eine ausreichende Klärung. Auf keinen Fall sollten verdünnte Seren filtriert werden, da vor allem IgM am Filtermaterial adsorbiert wird.

\section{Lösungsmittel}

Der Einfluß verschiedener Lösungsmittel auf Reaktionsgeschwindigkeit und Sedimentation der Antigen-Antikörper-Reaktion in der Laser-Nephelometrie wurde an den Beispielen IgG, IgA und IgM untersucht.

Die folgenden Diagramme zeigen die Reaktionskinetiken dreier verschiedener Verdünnungen des Standards in physiologischer $\mathrm{NaCl}$-Lösung und phosphatgepufferter $\mathrm{NaCl}-\mathrm{Lösung}$ am Beispiel von IgG (Abb. 3).

Man erkennt, daß die Geschwindigkeit der Reaktion in physiologischer $\mathrm{NaCl}$-Lösung größer ist als in phosphatgepufferter $\mathrm{NaCl}$-Lösung. Außerdem wurden in $\mathrm{NaCl}$ höhere Meßwerte erhalten. Bei hohen Antigenkonzentrationen ist die Reaktion bereits nach 15 Minuten zum größten Teil beendet, während bei mittleren und niedrigen Konzentrationen 30 Minuten und mehr benötigt werden. Bei hohen Konzentrationen wird das Meßsignal nach etwa 80 Minuten unruhig und steigt an. Dieser Effekt ist auf beginnende Sedimentation zurückzuführen. Die sich absetzenden Immunkomplexe verdich-

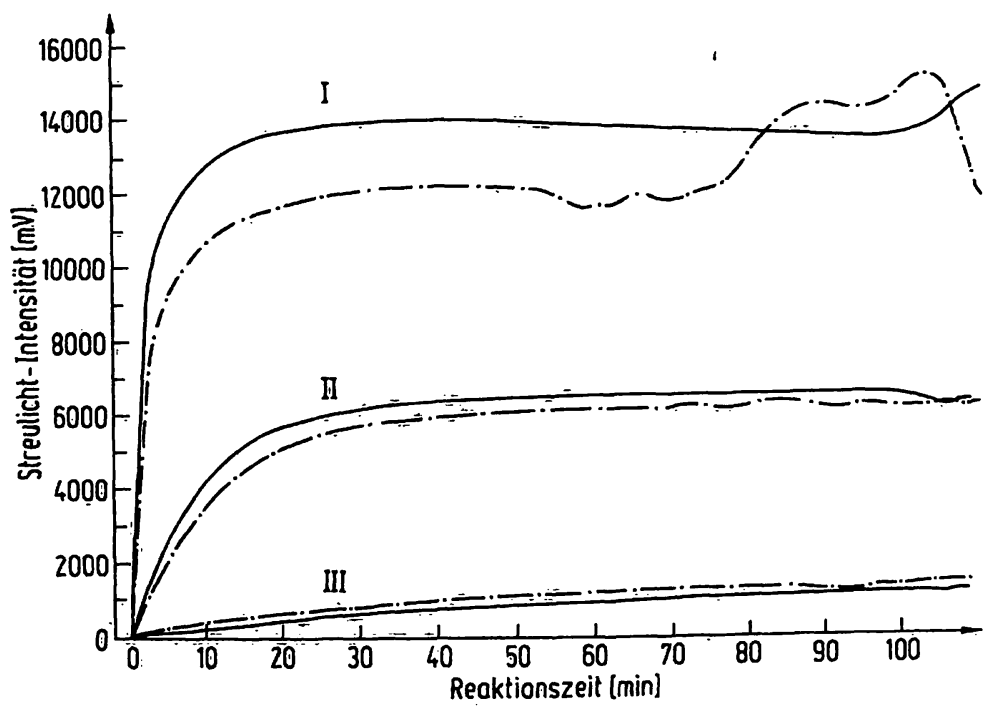

Abb. 3. Zeitlicher Verlauf der Reaktion in $\mathrm{NaCl}$ und phosphatgepufferter $\mathrm{NaCl}-\mathrm{Lösung} \mathrm{bei} \mathrm{IgG,} \mathrm{—} \mathrm{NaCl}, \cdot-\cdot-\cdot$ phosphatgepufferte $\mathrm{NaCl}, \mathrm{I}=0,524 \mathrm{~g} / 1, \mathrm{II}=0,131 \mathrm{~g} / \mathrm{l}, \mathrm{III}=0,016 \mathrm{~g} / \mathrm{l}$. 


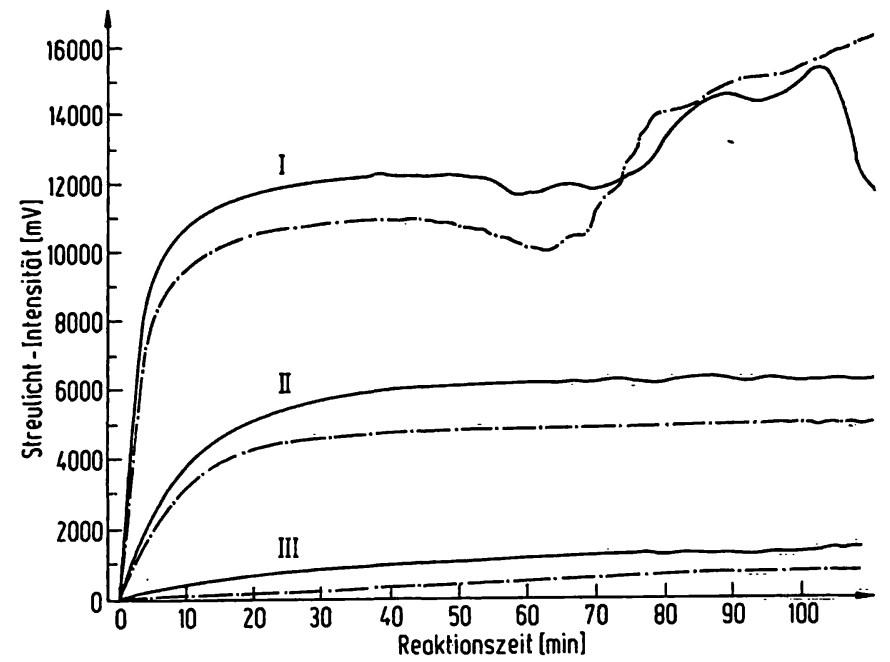

Abb. 4. Zeitlicher Verlauf der Reaktion in phosphatgepufferter $\mathrm{NaCl}$ mit und ohne Zusatz von Polyethylenglykol, - phosphatgepufferte $\mathrm{NaCl}$, .-.- phosphatgepufferte $\mathrm{NaCl} /$ Polyethylenglykol I $=0,524 \mathrm{~g} / \mathrm{l}, \mathrm{II}=0,131 \mathrm{~g} / \mathrm{l}, \mathrm{III}=0,016 \mathrm{~g} / \mathrm{l}$.

ten sich zunächst zu einer Wolke über dem Boden der Küvette, die in den Meßstrahl hineinragt, bevor sie vollständig auf den Boden absinkt. Wie aus Abbildung $3 \mathrm{zu}$ ersehen ist, tritt dieses Phänomen bei mittleren oder niedrigeren Konzentrationen erst nach Stunden ein, d. h. das Meßsignal ist über mehrere Stunden konstant.

Abbildung 4 zeigt die entsprechenden kinetischen Messungen mit $40 \mathrm{~g} / 1$ Polyethylenglykol-Zusatz zu phosphatgepufferter NaCl-Lösung. Man sieht, daß nach 30 bzw. 60 Minuten kein höheres Signal erhalten wird als in phosphatgepufferter $\mathrm{NaCl}$ ohne Zusatz. Die Sedimentation findet bei Polyethylenglykol-Zusatz sogar geringfügig früher statt als in $\mathrm{NaCl}$-Lösung.

\section{Reaktionszeiten}

Prinzipiell kann die Messung im kinetischen Bereich oder im Gleichgewichtszustand der Reaktion erfolgen. Die Ablesung nach 15 Minuten z. B. muß auf \pm 15 Sekunden genau erfolgen, was in einem Routinelabor bei einer größeren Anzahl von Messungen auf Schwierigkeiten stößt. Die Messung nach 1 Stunde kann mit einer Verzögerung von 15 Minuten erfolgen, ohne daß sich das Streulichtsignal wesentlich ändert.

Abbildung 5 zeigt Heidelberger-Kurven, die im LaserNephelometer nach 15 Minuten, 1 Stunde und 3 Stunden Reaktionszeit aufgenommen wurden.

Auch hier zeigt sich, daß 1 Stunde Reaktionszeit. günstiger ist, da sowohl die Empfindlichkeit als auch der Meßbereich größer werden.

\section{Meßbereiche}

Das Laser-Nephelometer ist in der Lage, einen großen Meßbereich stufenlos zu überstreichen. So sind IgG in einem Bereich von 17-1.150 IU/ml (1,5-100 g/l), IgA

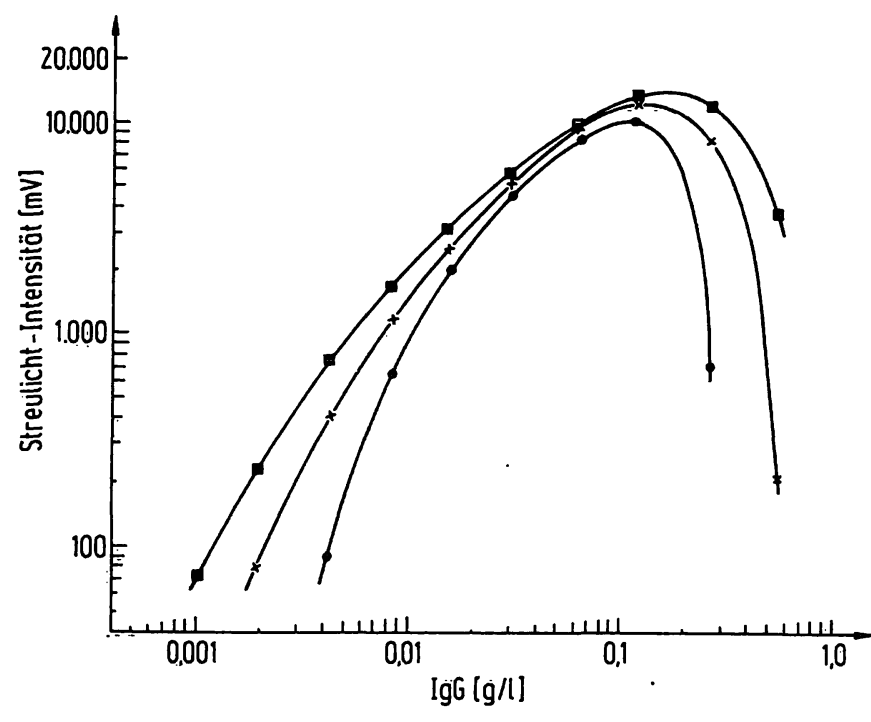

Abb. 5. Referenžkurven für die IgG-Bestimmung bei 15 Minuten $(\bullet-\bullet), 1$ Stunde $(x=-x)$ und 3 Stunden $(a--)$ Reaktionszeit.

von $36-595 \mathrm{IU} / \mathrm{ml}(0,6-10 \mathrm{~g} / \mathrm{l})$ und $\mathrm{IgM}$ von $36-1.150$ $\mathrm{IU} / \mathrm{ml}(0,3-10 \mathrm{~g} / 1)$ meßbar, wenn man nach dem Verdünnungsschema von Tabelle 1 arbeitet. Diese Meßbereiche umfassen sowohl den gesamten Normalbereich als auch größere Teile des pathologischen Bereichs. Bei . Konzentrationen oberhalb des Meßbereiches können die Seren höher verdünnt oder in kleineren Volumina, z. B. $50 \mu$ statt $100 \mu \mathrm{l}$ bei Ig $\dot{A}$ oder IgM, eingesetzt werden. Konzentrationen, die unterhalb des Meßbereiches liegen, können bis zu einer gewissen Grenze durch niedrigere Serumverdünnungen erfaßt werden. Diese Grenze ist durch die untere Nachweisgrenze gegeben, die von der Eigentrübung des Untersuchungsmaterials abhängt.

Unter den untersuchten Seren war keines, das von dem angegebenen Meßbereich nicht erfaßt wurde, was ein Hinweis dafür ist, daß die Bereiche für Routineuntersuchungen ausreichend sind.

\section{Antigenüberschuß}

Das Digitalvoltmeter des Laser-Nephelometers zeigt keine Streulichtsignale an, die mehr als $19,99 \mathrm{~V}$ betragen, d. h. die Anzeige erlischt.

Bei der IgG- und IgA-Bestimmung bedeutet das, daß die Maxima der entsprechẹnden Heidelberger-Kurven oberhalb $20 \mathrm{~V}$ liegen. Die Gefahr von Antigenüberschuß entsteht erst dann, wenn das Meßsignal, das zum rechten absteigenden Ast der Heidelberger-Kurve gehört, unter $20 \mathrm{~V}$ liegt.

Bei IgG ist dieser Punkt bei $350 \mathrm{~g} / 1$ und bei IgA bei etwa $40 \mathrm{~g} / \mathrm{l}$ erreicht. Das Maximum der IgM-Kurve liegt unterhalb von 20 V. Die Konzentration im Maximum beträgt etwa $40 \mathrm{~g} / 1$. 


\section{Präzision}

Um die Präzision der Laser-Nephelometrie in der Serie und von Tag zu Tag zu überprüfen, wurden Doppelbestimmungen von einer Vielzahl von Seren statistisch ausgewertet.

Die folgenden Abbildungen zeigen, daß sowohl in der Serie (Abb. 6) als auch von Tag zu Tag (Abb. 7) eine gute Ubereinstimmung der Ergebnisse besteht.

Tabelle 3 faßt die dabei gefundenen Variationskoeffizienten zusammen.

\section{Korrelation zur radialen Immundiffusion}

Die folgende Tabelle (Tab. 4) gibt die beim statistischen Vergleich zwischen radialer Immundiffusion und LaserNephelometrie gefundenen Kenndaten für die Korrelation wieder.

Die folgende Abbildung (Abb. 8) zeigt graphisch, daß eine gute Úbereinstimmung beider Methoden bei der quantitativen Bestimmung der Immunglobuline G, A und $M$ besteht.

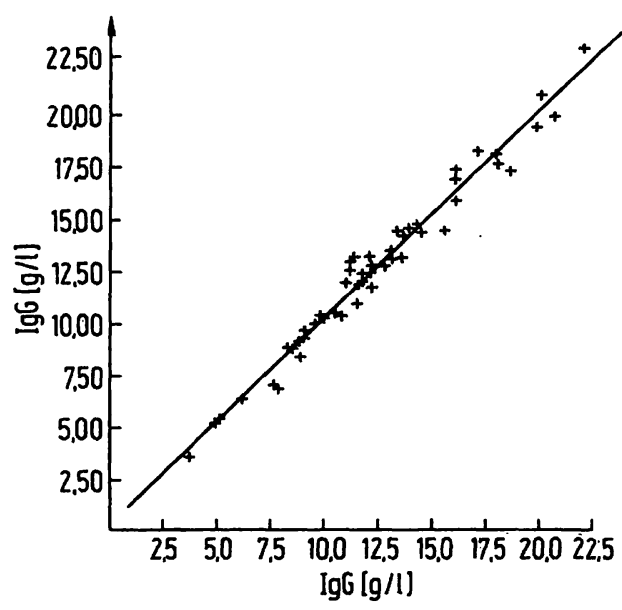

Abb. 6. Präzision in der Serie

a) bei IgG $y=0,992 x+19,230$
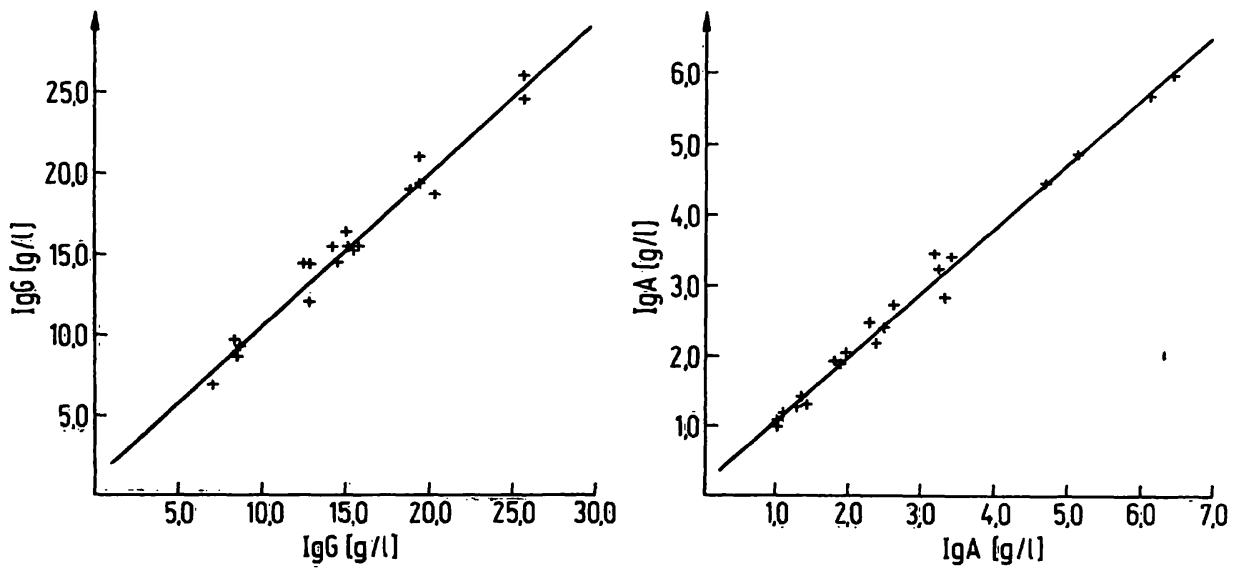

b) bei IgA $y=0,919 x+15,384$

Abb. 7. Präzision von Tag zu Tag

a) bei. $\operatorname{Ig} \dot{G} \quad y \equiv 0,950 \bar{x}+105,707$

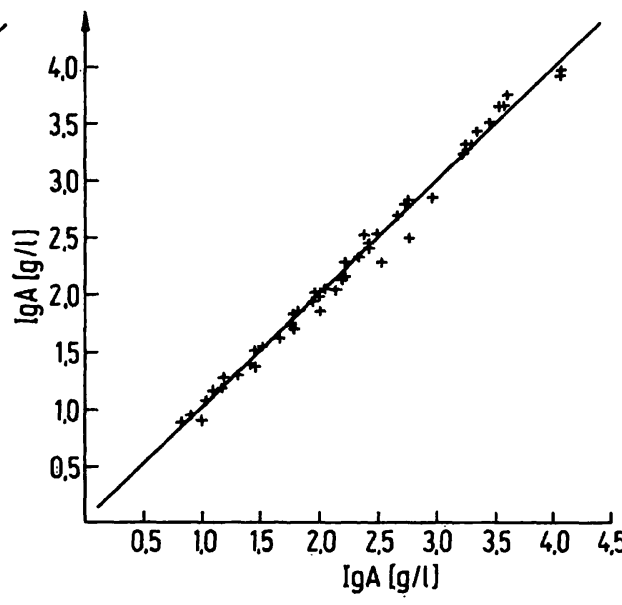

b) bei IgA $y=0,999 x+1,792$

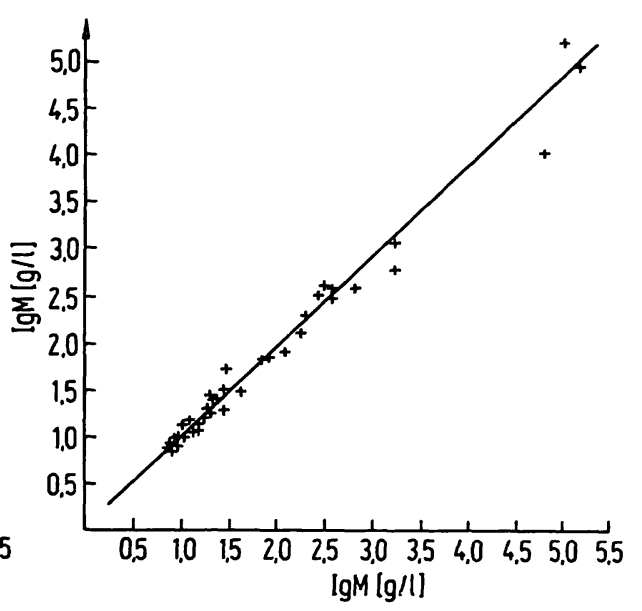

c) bei IgM $y=0,962 x+3,040$

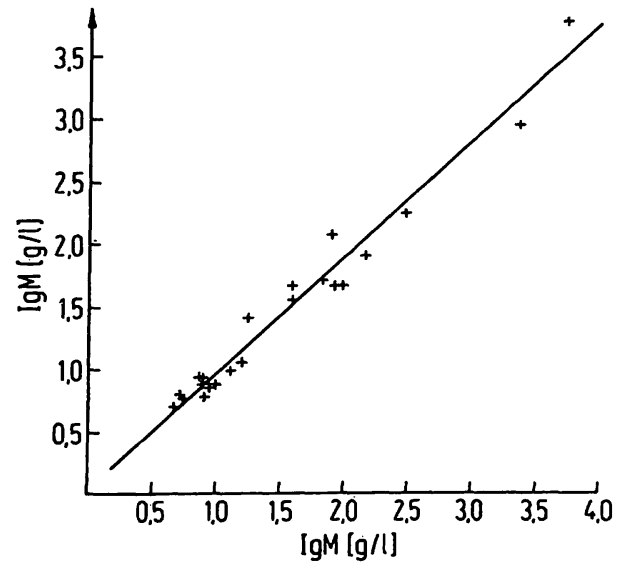

c) bei IgM $y=0,929 x+3,703$

Tab. 3. Mittelwerte, Standardabweichung und Variationskoeffizienten bei Unterssuchungen der Präzision in der Laser-Nephelometrie

\begin{tabular}{|c|c|c|c|c|c|c|c|c|}
\hline & \multicolumn{4}{|c|}{ Präzision in der Serie } & \multicolumn{4}{|c|}{ Präžision von Tag zu Tag } \\
\hline & $\overline{\mathbf{x}} \cdot[\mathrm{g} / \mathrm{l}]$ & s. $[g / 1]$ & VK [\%] & $\mathbf{n}$ & $\bar{x}[g / l]$ & $s[g / 1]$ & VK $[\%]$ & $\mathrm{n}$ \\
\hline $\begin{array}{l}\text { IgG } \\
\text { IgA } \\
\text { IgM }\end{array}$ & $\begin{array}{r}12,66 \\
2,15 \\
1,60\end{array}$ & $\begin{array}{l}0,67 \\
0,09 \\
0,11\end{array}$ & $\begin{array}{l}3,8 \\
3,2 \\
5,4\end{array}$ & $\begin{array}{l}54 \\
55 \\
48\end{array}$ & $\begin{array}{r}15,19 \\
2,63 \\
1,49\end{array}$ & $\begin{array}{l}0,67 \\
0,15 \\
0,12\end{array}$ & $\begin{array}{l}4,4 \\
5,6 \\
8,3\end{array}$ & $\begin{array}{l}22 \\
22 \\
22\end{array}$ \\
\hline
\end{tabular}



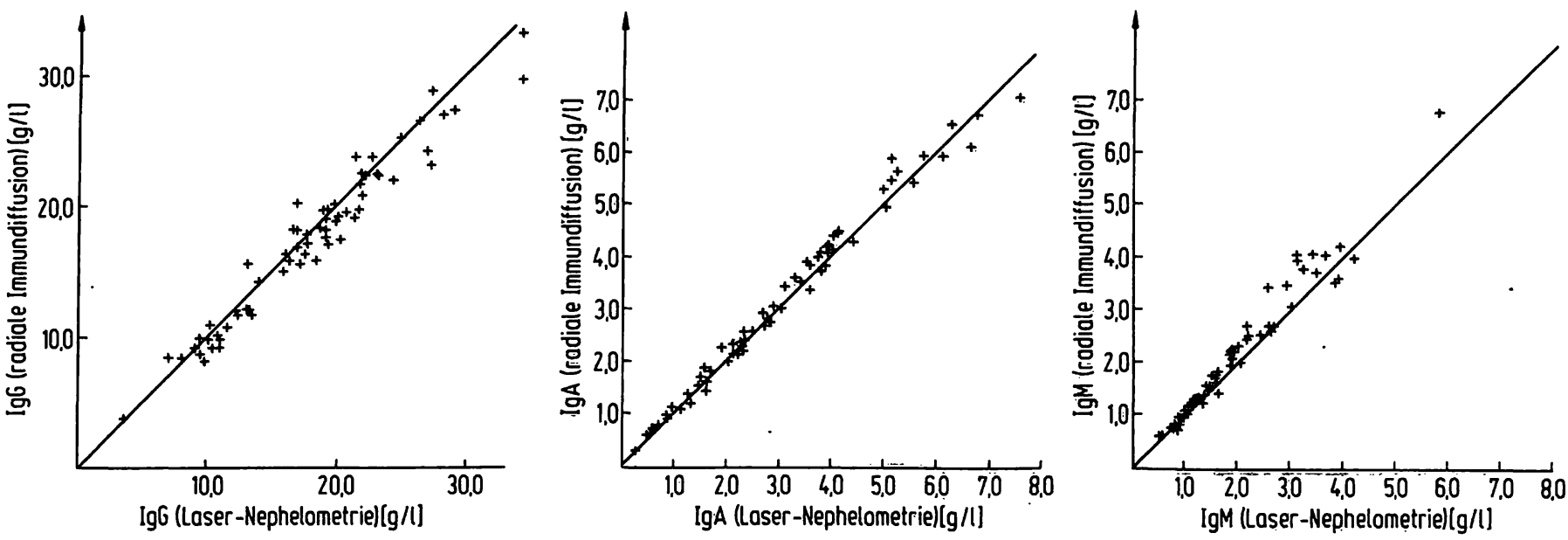

Abb. 8. Korrelation zwischen radialer Immundiffusion und Laser-Nephelometrie
a) bei IgG
b) bei $\operatorname{Ig} \mathbf{A}$
c) bei IgM

Tab. 4. Statistischer Vergleich von radialer Immundiffusion und Laser-Nephelometrie

\begin{tabular}{llll}
\hline & IgG & IgA & IgM \\
\hline Steigung der Regressionsgeraden & 0,986 & 0,937 & 0,840 \\
Schnittpunkt x-Achse [g/1] & 0,982 & 0,079 & 0,168 \\
Korrelationskoeffizient $\mathrm{r}$ & 0,975 & 0,981 & 0,967 \\
\hline
\end{tabular}

Es fällt auf, daß bei IgM ab einer Konzentration von etwa $2,5 \mathrm{~g} / 1$ eine Parallelverschiebung zu niedrigeren Werten in der Laser-Nephelometrie eintritt.

\section{Diskussion}

\section{Blindwerte}

Bei nephelometrischen Proteinbestimmungen läßt sich das gesamte Streulichtsignal, das man bei der Messung der Antigen-Antikörper-Reaktion erhält, in verschiedene Anteile zerlegen, die nicht auf Streuung des Lichtes an Immunkomplexen zurückzufüren sind. Es sind dies die Komponenten des Streulichtes, die verursacht werden durch die Küvetten, durch die Eigentrübung des Verdünnungsmittels, des Antiserums und der Proben. Diese Komponenten gehen unterschiedlich stark in die Gesamtstreulichtintensität ein, wobei die Eigentrübung der untersuchten Patientenseren die größte Komponente mit den größten Schwankungen von Probe zu Probe darstellt.

Der Anteil der Streulichtsignale durch Puffer und Antiserumverdünnung ist sowohl bei den Standardproben als auch bei den zu untersuchenden Seren gleich. Deswegen wäre es sinnlos und überflüssige Arbeit, wenn man diese konstanten Werte sowohl von der Referenzkurve, was nur eine Parallelverschiebung verursachen wïrde, als auch von den Probenmeßwerten subtrahieren würde.
Bei den bisher bekannten nephelometrischen Meßmethoden wird ohne nähere Begründung davon ausgegangen, daß sämtliche Komponenten additiv in das Gesamtstreulichtsignal eingehen, d. h. daß man durch einfache Subtraktion der einzelnèn Blindwerte zu dem eigentlichen Streulichtsignal gelangt, das durch die Immunkomplexe verursacht wird. Ein Beweis für diese Annahme wurde bisher nicht erbracht. Unsere Experimente deuten darauf hin, daß dieser additive Zusammenhang besonders bei Proben mit starker Eigentrübung nicht besteht.

Möglicherweise findet eine Aggregatbildung zwischen den entstehenden Antigen-Antikörper-Komplexen und den übrigen Trübungspartikeln in der Probe statt, so daß keine reine Summation von Teilchen vorliegt.

Für die Praxis empfiehlt es sich daher, bei der nephelometrischen Proteinbestimmung von möglichst klarem Untersuchungsmaterial auszugehen, z. B. nüchtern entnommene Seren. In der Laser-Nephelometrie ist bei klaren oder leicht trüben Seren wegen des großen Unterschiedes von Meßsignal zu Untergrund eine Blindwertkorrektur nicht nötig. Stark trübe Proben sollten geklärt werden.

\section{Reaktionszeiten}

Sowohl die Auswertung nach 15 Minuten mit zeitlich exakter Ablesung als auch nach einer Stunde führt zu genauen Meßergebnissen. Bei der vorzeitigen Ablesung sind die Meßbereiche kleiner und dadurch die unteren Nachweisgrenzen unempfindlicher. Nach einer Stunde findet noch keine Sedimentation der Immunkomplexe statt. Diese fängt bei den höchsten Konzentrationen nach etwa 75-80 Minuten an.

\section{Lösungsmittèl}

Die Messungen erfolgen in physiologischer $\mathrm{NaCl}$-Lösung. Andere Reaktionsmedien zeigen keine Vorteile. Da die 
Antigen-Antikörper-Reaktionen in weiten Bereichen vom pH-Wert unabhängig sind (4), besteht auch keine Notwendigkeit, zu gepufferten Systemen überzugehen. In phosphatgepufferter $\mathrm{NaCl}$-Lösung tritt sogar eine Verzögerung der Reaktion ein. Die Meßsignale liegen unter denen, die in $\mathrm{NaCl}$-Lösung erhalten werden.

Der Zusatz von Polyethylenglykol bringt bei der Bestimmung der Immunglobuline $\mathbf{G}, \mathbf{A}$ und $\mathrm{M}$ keine Vorteile, da die Reaktion mit oder ohne das Reagenz nach einer Stunde beendet ist. Eine Stabilisierung der Immunkomplexe in der Lösung konnte weder durch Polyethylenglykol noch durch andere Zusätze wie Tween 20 oder Triton X 100 erreicht werden.

\section{Meßbereiche}

Die Meßbereiche sind in den beschriebenen Experimenten so breit, daß die meisten im klinischen Labor anfallenden Proben von ihnen erfaßt werden. Zusätzlich besteht bei IgG und IgA nach oben eine Sicherheitszone, die auf den absteigenden Ast der Heidelberger-Kurve übergreift, bei der das Streulichtsignal oberhalb von $20 \mathrm{~V}$ liegt.

Die Meßbereiche sind so gewählt, daß in der Nähe des Maximums der Heidelberger-Kurve nicht mehr gearbeitet wird, da wegen des Abflachens der Kurve die Ablesefehler zu groß werden. Deswegen liegt die obere Grenze des IgM-Meßbereiches bei etwa 10 g/l, obwohl der Gipfel der Kurve bei etwa $40 \mathrm{~g} / 1$ liegt. Wegen der Breite dieser Bereiche dürfte ein Antigenüberschuß in der Praxis selten vorkommen.

\section{Literatur}

1. Sieber, A. \& Gross, J. (1975), Protides Biol. Fluids, Proc. Colloq. 23, 295-298.

2. Sieber, A. \& Gross, J. (1976), Laboratoriumsblätter Behringwerke AG 26, 117-123.

3. Deaton, C. D. \& Kameron, W. (1976), Clin. Chem. 22, $1465-1471$.

\section{Präzision}

Die Präzision der radialen Immundiffusion ist in verschiedenen Arbeiten untersucht worden (5). Danach beträgt der Variationskoeffizient etwa 3-7\%.

Die Ergebnisse mit dem Laser-Nephelometer beweisen, daß die Methode zu ebenso exakten Ergebnisse führt. Zweifellos sind die Resultate noch zu verbessern, wenn die Methode automatisiert wird und somit Verdünnungsund Dosierfehler weitgehend ausgeschlossen werden können.

Korrelation $\mathrm{zw}$ ischen radialer Immundiffusion und Laser-Nephelometrie

Die klinische Praxis hat gezeigt, daß eine sehr gute Úbereinstimmung zwischen radialer Immundiffusion und Laser-Nephelometrie besteht.

Der Umstand, daß bei IgM ab einer Konzentration von etwa 2,5 g/l eine Parallelverschiebung der Meßwerte eintritt (s. Abb. 8c) fällt mit der Tatsache zusammen, daß ab dieser Konzentration die Seren bei der PartigenMethode verdünnt werden müssen. Offenbar verhält sich IgM im verdünnten Zustand bei der Immundiffusion anders als im natürlichen Serumverband. Möglicherweise spielen nur Veränderungen der Molekülgröße eine Rolle.

Die experimentellen Daten beweisen, daß die LaserNephelometrie eine schnelle, einfache und zuverlässige Methode zur quantitativen Proteinbestimmung darstellt. Möglicherweise wird sich die Methode auch auf andere Gebiete übertragen lassen, wo Reaktionen stattfinden, bei denen Trübungen entstehen oder verschwinden.

4. Killingsworth, L. M. \& Savory, J. (1973), Clin. Chem. 19, 403-407.

5. Lamerz, R. \& Fateh-Moghadam, A. \& Knedel, M. (1973), diese Z. 11, 491-500.

Dr. A. Sieber Behringwerke AG D-3550 Marburg/Lahn 
